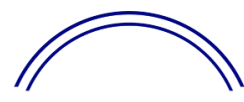

\title{
A Cross-Sectional Survey of Patients and Staff on Inpatient Forensic Psychiatric Units in Canada During the COVID-19 Outbreak
}

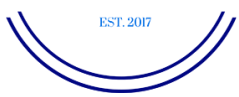

\author{
Courtney Brennan, ${ }^{1,2}$ Dana Moyer, ${ }^{2}$ Sabrina Umbrello, ${ }^{2}$ Patti Socha, ${ }^{1}$ \\ Alexander Simpson, ${ }^{1,3}$ Sean A. Kidd ${ }^{1,3}$
}

${ }^{1}$ Centre for Addiction and Mental Health, Toronto, Canada; ${ }^{2}$ Department of Occupational Science and Occupational Therapy, University of Toronto, Toronto, Canada; ${ }^{3}$ Department of Psychiatry, University of Toronto, Toronto, Canada

\begin{abstract}
Outbreaks of the coronavirus disease (COVID-19) on inpatient forensic psychiatry units present a unique challenge as early release is not possible and some facilities were not designed to achieve sustained social distancing. The enforcement of droplet and contact (D\&C) precautions required by Public Health Ontario during an outbreak creates further confines and restrictions for patients that are typically subject to considerable constraints during their care. From December 2020 to January 2021, 30 clinicians and 12 patients on inpatient forensic psychiatry units under unit-wide D\&C precautions during COVID-19 outbreaks completed a cross-sectional survey about their experience. We also conducted virtual focus groups to triangulate the qualitative feedback from clinicians. The survey and focus group discussions revealed similar themes of enablers, barriers, and desired changes to care provision during an outbreak. We discuss findings within the broader context of outbreak interventions and the provision of services to those living and working on forensic psychiatry inpatient units experiencing outbreaks requiring the unit-wide implementation of D\&C precautions.
\end{abstract}

Keywords: COVID-19, schizophrenia, inpatient, forensic, isolation, outbreak

\section{Introduction}

Individuals confined to secure psychiatric environments are more vulnerable to the coronavirus disease (COVID-19) due to their physical and psychiatric comorbidities, the challenges of early release, and facilities and inpatient environments often not being designed to achieve sustained social distancing $[1,2]$. While necessary to protect the public, the restrictions placed on patients in forensic inpatient environments also bring negative consequences, including violent events and deterioration of mental status [3], poor ward atmosphere, and patient experiences of punitiveness and violation of dignity [4].
The enforcement of droplet and contact (D\&C) precautions are implemented unit-wide for 14 days when a public health COVID-19 outbreak is declared (i.e., two or more infected patients) [5]. For staff, D\&C precautions include enhanced hand hygiene, increased use of personal protective equipment (PPE) (e.g., mask, eye protection, gown, gloves), and performance of enhanced cleaning tasks. D\&C precautions needed during an infectious outbreak create further confines and restrictions for patients who are typically subject to considerable constraints during their care. For clients, D\&C precautions include isolating to their rooms on the unit, monitored use 
of shared washrooms with enhanced cleaning between uses, mask compliance in all shared spaces of the unit, and a hold on all off-unit visitors (e.g., family, food deliveries). If a patient is unable to abide by isolation practices reasonably or safely, hospital policy required all reasonable attempts to be made to use alternative, least restrictive, or least intrusive strategies before using seclusion or restraint to limit the risk of exposure or spread.

The increase in restrictions and limitations associated with D\&C precautions poses ethical concerns about additional restrictions of liberties placed on patients already confined. Reviews of the literature [1,2] identify changes to care delivery and outbreak management strategies for units on D\&C precautions. However, little is known about the acceptability of these interventions to patients and staff, or the impact on the mental health and wellbeing of the people living and working on units during an outbreak. In this exploratory study, we surveyed patients and staff from two minimumsecurity forensic psychiatry inpatient units to gain insight into the experience of living and working in these environments under D\&C precautions during the COVID-19 pandemic and develop responses to these experiences.

\section{Methods}

\section{Design}

For this cross-sectional study, we recruited patients and staff on two inpatient forensic psychiatry units (a total of 40 beds) that had experienced a COVID-19 outbreak at the Centre for Addiction and Mental Health (CAMH). The study involved the use of both quantitative and qualitative methodologies obtained through surveys and focus groups.

\section{Participants}

All patients and staff on two inpatient minimum secure forensic units experiencing outbreaks were invited to participate in the survey about one-month postoutbreak. CAMH has 100 minimum secure forensic beds located in one out-of-date tower. These units have a small footprint, limited bedroom and lounge space, and shared toilet facilities. Units are highly staffed (daily staff to patient ratio is about $1: 1.8$ ) and staff accommodation is cramped.

Most patients have a primary diagnosis of a psychotic disorder. Neurodevelopmental and degenerative mental disorders are common comorbidities. These conditions gave rise to the heightened risk of COVID-19 outbreaks [1]. At the time of our study, patients were all found not criminally responsible on the grounds of mental disorder. We surveyed the two minimum secure units that had experience recent COVID-19 outbreaks A total of 40 patients were invited to participate. Participation in the survey and focus groups was not mutually exclusive. Patients were invited to participate in-person on the unit and given the option to complete the survey independently or facilitated to complete surveys at their request by a clinician external to their unit not involved in their care.

Clinicians were invited to participate via email.

\section{Data collection}

Quantitative and qualitative data were obtained through patient and staff surveys as well as staff focus groups. Both the surveys and focus groups contained a mix of closed- and openended questions created specifically for this study. Surveys were anonymously conducted online through REDCap and took about 15 minutes to complete the patient survey consisted of nine questions exploring the attitudes, feelings, overall well-being, and quality of life experienced while residing on the unit during a COVID-19 outbreak. The staff survey consisted of 10 questions exploring the experiences and perspectives of delivering care to forensic inpatients during an outbreak and under D\&C precautions. In addition to the survey, clinicians from both Unit A and Unit B were invited to participate in one of three focus groups that further explored their experiences in providing patient care, as well as barriers they experienced during a COVID-19 outbreak. Focus group data were used to triangulate 
Table 1: Methods of data analysis

Stages 1 and 2: Coding comments about decreased programming and combining the statements

\begin{tabular}{lll}
\hline Comments & Coded comments & Subcategories \\
\hline "Couldn't do anything" (PSR) & Limited programs available to clients & Decreased \\
"Boring" (PSR) & resulted in increased boredom & $\begin{array}{l}\text { programming being } \\
\text { offered on and off the }\end{array}$ \\
"Most of the group programs in and off the & $\begin{array}{l}\text { Clear reduction in the number of } \\
\text { programs offered on the unit }\end{array}$ & unit \\
unit has been cancelled" (CSR) & P
\end{tabular}

Regarding interventions, "a lot are in comfort room, which is shared; not able to use during outbreak because clients were in their rooms and it would involve a lot of cleaning" (occupational therapist, CFG)

"A lot of interventions listed we don't have enough to give a single one to each client" (nurse, CFG)

"Less activity/programs, if there is a program, it will be restricted" (CSR)

“We couldn't run any in-person groups and taking on the virtual groups was challenging. Types of groups we are able to provide are not the same as we did, and it's met with a lot of disappointment from clients" (recreation therapy, CFG)

"It was hard to not even go to the yard if you don't have COVID you shouldn't be penalized like that" (PSR)

"We have a client on the unit who hasn't been in the fresh air in months" (occupational therapy, CFG)

"Limited access to fresh air" (CSR)

"Unable to get fresh air" (CSR)

"Clients not being able to access fresh air because they aren't able to wear a mask properly is a bit of an issue" (occupational therapist, CFG)

"Decreased physical activity and engagement in therapeutic groups" (CSR)

Lack of resources needed to run programs for everyone on the unit

Programs that were running were subjected to safety restrictions

Limited access to outdoor activities

Restrictions in programming were sometimes a result of an inability to adhere to D\&C precautions

There was a decrease in physical activity

"Physical activity limitations" (CSR)

Stage 3: Combining subcategories with similar content into generic categories

\begin{tabular}{ll}
\hline Subcategories & Generic categories \\
\hline Change in living conditions & Decreased well-being \\
The need for isolation of clients in their rooms & and quality of life of \\
Decreased programming being offered on the unit & clients on the units \\
Decreased socialization among clients and with unit staff & \\
Restricted outside services into the unit (e.g., online shopping, ordering food) & \\
Paused progression to discharge into the community & \\
\hline
\end{tabular}


Table 1 continued

Stage 4: Combining the generic categories into main categories

\begin{tabular}{ll}
\hline Generic categories & Main categories \\
\hline Decreased well-being and quality of life of clients on the unit & Barriers to quality of \\
Droplet and contact (D\&C) precautions & $\begin{array}{l}\text { caring COVID-19 } \\
\text { outbreak precautions on } \\
\text { fension between clinical staff, as well as between clients and clinicians } \\
\text { Limited resources experienced by clients and clinicians }\end{array}$ \\
Leadership barriers & Supportive aspects \\
More time spent in room allowing participation in individual activities & of quality care during \\
Patients enjoyed access to electronic devices and services & COVID-19 outbreak \\
Supportive team dynamics & precautions on forensic \\
Dedication to safety of both clients and clinicians & units \\
Promote client well-being and quality of life & Changes desired to \\
Improved access to and cleanliness of bathrooms on the unit & improve quality of \\
Increased access to resources for clinicians and clients & care in future outbreak \\
Proactive leadership from the organization & precautions on forensic \\
\end{tabular}

Note: $P S R=$ patient survey response, $C S R=$ clinician survey response, $C F G=$ clinician focus group

survey data. Focus groups lasted between 30 and 90 minutes. We recorded audio to ensure accuracy.

\section{Data analysis}

We began data analysis by listening to audio recordings of focus groups and transcribing them verbatim as well as extracting written comments from the surveys. Next, an inductive content analysis [6] was performed by two researchers (D. Moyer and S. Umbrello). First, quotations were coded and similar statements were grouped to form subcategories. The subcategories were then combined with other similar contents into generic categories. Lastly, the generic categories were combined to create main categories or themes. An example can be seen in Table 1.

\section{Ethics}

According to the policy activities that constitute research at $\mathrm{CAMH}$, this project met the criteria for operational improvement activities. Study design and data collection for patients and staff was approved through the institutional quality performance ethics review process. All participants provided informed consent.

\section{Results}

\section{Sample characteristics}

Survey responses were obtained from 12 patients (Unit $A=5$, Unit $B=7$, response rate $=$ $32.4 \%$ ) and 30 staff (Unit $A=11$, Unit $B=19$, response rate $=38.45 \%$ ). Seven staff participated in focus groups. People from various professional disciplines participated in the survey and focus groups, including nursing $(14,46.7 \%)$, recreation therapy $(3,10.0 \%)$, behaviour therapy $(3,10.0 \%)$, occupational therapy $(2,6.7 \%)$, program assistant $(2,6.7 \%)$, other clinician (5, $16.7 \%)$ and unspecified (1,3.3\%). Quantitative survey results can be found in Tables 2 and 3 .

\section{Qualitative results}

While coding qualitative client and clinician survey and focus group responses, three main themes emerged:

1. barriers to quality care,

2. supportive aspects of care, and

3. suggestions for changes to improve the quality of care in the event of a future outbreak. 
Table 2: Clinician quantitative survey results, $n=30$

Question
How do you feel the COVID droplet and contact
precautions have impacted, if at all, your clients'
quality of life on the unit?

Results, $\boldsymbol{n}(\%)$

$16(55.2 \%)$ COVID precautions both positively and negatively

influenced individual client quality of life on the unit

$11(37.9 \%)$ COVID precautions negatively influenced client quality of life on the unit

$2(6.9 \%)$ COVID precautions positively influenced client quality of life on the unit

$1(3.3 \%)$ Did not respond

What specific responses did you see from clients? $24(80.0 \%)$ Increase in requests to staff

$20(66.7 \%)$ Stayed in room without issue

19 (63.3\%) Neglected ADLs (activities of daily living)

$15(50.0 \%)$ Required seclusion

$15(50.0 \%)$ Bizarre behaviours

$14(46.7 \%)$ Increased behaviours

$7(23.3 \%)$ Decreased behaviours

$6(20.0 \%)$ Other

$5(16.7 \%)$ Violent outbursts

$5(16.7 \%)$ No specific behaviours observed

$4(13.3 \%)$ Increased enjoyment

$1(3.3 \%)$ Improved activities of daily living

Did client responses evolve throughout

implementing the restrictions?

$26(89.7 \%)$ Yes

$3(10.3 \%)$ No

$1(3.3 \%)$ Did not respond

Did you feel supported during the COVID droplet and contact precautions?

$17(56.7 \%)$ Yes

$9(30.0 \%)$ Somewhat

$4(13.3 \%)$ No

What was hard about the COVID droplet and contact precautions?

$24(80.0 \%)$ Client isolation

$23(76.7 \%)$ Client well-being

$22(73.3 \%)$ Maintaining D\&C (droplet and contact) precautions

$17(56.7 \%)$ Limited client contact

$16(53.3 \%)$ Insufficient staffing

$14(46.7 \%)$ Alternative programming

$12(40.0 \%)$ Logistics of virtual care

$8(26.7 \%)$ Insufficient PPE (personal protective equipment)

$7(23.3 \%)$ Other (please specify)

What was easy about the COVID droplet and contact precautions?

$18(72.0 \%)$ Sufficient PPE

$5(20.0 \%)$ Sufficient staffing

$5(20.0 \%)$ Client contact

$3(12.0 \%)$ Virtual care

$3(12.0 \%)$ Client isolation

$3(12.0 \%)$ Other (please specify)

$2(8.0 \%)$ Client well-being

$2(8.0 \%)$ Programming

$2(8.0 \%)$ Maintaining D\&C precautions

How do you feel the COVID-19 droplet and contact precautions influenced the relationship

$18(60.0 \%)$ Mixed impact

7 (23.3\%) No impact

between clients and clinicians on the unit?

$4(13.3 \%)$ Negative impact

$1(3.3 \%)$ Positive impact

Did the precautions contribute to any potential

$17(56.7 \%)$ No

$13(43.3 \%)$ Yes

$1(3.3 \%)$ Did not respond

continued 
Table 2 continued

\begin{tabular}{|c|c|}
\hline Question & Results, $n$ (\%) \\
\hline $\begin{array}{l}\text { What barriers did you come across in your } \\
\text { practice? }\end{array}$ & $\begin{array}{l}24(80.0 \%) \text { Emotional exhaustion/burnout } \\
16(53.3 \%) \text { Insufficient staffing } \\
12(40.0 \%) \text { Alternative intervention delivery } \\
3(10.0 \%) \text { Other (please specify) }\end{array}$ \\
\hline $\begin{array}{l}\text { Do the restrictions need to be adapted to better } \\
\text { meet the needs of clients within an inpatient } \\
\text { forensic practice setting? }\end{array}$ & $\begin{array}{l}14(48.3 \%) \text { Yes } \\
15(51.7 \%) \text { No } \\
1(3.3 \%) \text { Did not respond }\end{array}$ \\
\hline How did you feel during the outbreak on the unit? & $\begin{array}{l}25(83.3 \%) \text { Stressed } \\
23(76.7 \%) \text { Tired } \\
22(73.3 \%) \text { Anxious } \\
18(60.0 \%) \text { Overwhelmed } \\
12(40.0 \%) \text { Nervous/scared } \\
5(16.7 \%) \text { Other } \\
4(13.3 \%) \text { Neutral }\end{array}$ \\
\hline What coping strategies did you use, if any? & $\begin{array}{l}20(71.4 \%) \text { Asking for support when needed } \\
20(71.4 \%) \text { Self-care } \\
13(46.4 \%) \text { Relaxation/meditation } \\
13(46.4 \%) \text { Time management } \\
13(46.4 \%) \text { Creating boundaries } \\
10(35.7 \%) \text { Mindfulness } \\
8(28.6 \%) \text { Social time with others } \\
7(25.0 \%) \text { Physical activity } \\
3(10.7 \%) \text { Leisure activities } \\
2(7.1 \%) \text { Other (please specify) }\end{array}$ \\
\hline
\end{tabular}

The qualitative results are summarized in Table 4.

\section{Barriers to quality of care}

The main barriers to patient care were grouped into the following five generic categories:

1. decreased well-being and quality of life of patients on the unit,

2. D\&C precautions,

3. tension between clinical staff, as well as between patients and clinicians,

4. limited resources experienced by patients and clinicians, and

5. leadership challenges.

One of the barriers affecting the decreased well-being and quality of life of patients on the units was the change in living conditions and the need to isolate patients. Many of the patients were restricted to their rooms, unable to access the common areas on the unit during lockdown periods. These changes were a big adjustment for the patients on the units. One clinician stated, "clients found it difficult to adjust to staying in their rooms for a long period of time" (survey). The changes in their usual dayto-day structure undoubtedly had an impact on patient well-being, as one patient stated they "felt lonely" (survey). The changes were also clearly observed by staff working on the unit, with clinicians stating, "Clients became more isolated in their room and more depressed" (survey). "Being confined to their rooms for 14+ days was challenging for some" (survey).

In analyzing the survey responses, one of the biggest barriers affecting the overall well-being of patients was the decrease in programming being offered. As a result of the restrictions and the need to protect the health and safety of patients and clinicians, many of the programs 
Table 3: Patient quantitative survey results, $n=12$

\begin{tabular}{|c|c|}
\hline Question & Results, $n(\%)$ \\
\hline $\begin{array}{l}\text { How do you feel the COVID droplet and contact } \\
\text { precautions have impacted, if at all, your quality of } \\
\text { life on the unit? }\end{array}$ & $\begin{array}{l}5(45.5 \%) \text { COVID precautions both positively and negatively } \\
\text { influenced individual client quality of life on the unit } \\
4(36.4 \%) \text { COVID precautions negatively influenced client quality } \\
\text { of life on the unit } \\
2 \text { (18.2) COVID precautions positively influenced client quality of } \\
\text { life on the unit } \\
1(8.3 \%) \text { Did not respond }\end{array}$ \\
\hline $\begin{array}{l}\text { What things did you want to do that you weren't } \\
\text { able to because of the COVID droplet and contact } \\
\text { precautions? }\end{array}$ & $\begin{array}{l}10(83.3 \%) \text { Go outside } \\
7(58.3 \%) \text { Other (please specify) } \\
5(41.7 \%) \text { Socialize with others } \\
5(41.7 \%) \text { Have visitors } \\
3(25.0 \%) \text { Group meals } \\
2(16.7 \%) \text { In-person groups }\end{array}$ \\
\hline How did you feel during the outbreak on the unit? & $\begin{array}{l}9(75.0 \%) \text { Other (please specify) } \\
5(41.7 \%) \text { Lonely } \\
4(33.3 \%) \text { Sad } \\
3(25.0 \%) \text { Tired } \\
2(16.7 \%) \text { Angry } \\
2(16.7 \%) \text { Scared } \\
2(16.7 \%) \text { Neutral } \\
1(8.3 \%) \text { Happy }\end{array}$ \\
\hline $\begin{array}{l}\text { What was hard about the COVID droplet and } \\
\text { contact precautions? }\end{array}$ & $\begin{array}{l}9(75.0 \%) \text { Staying in my room } \\
7(58.3 \%) \text { Other (please specify) } \\
6(50.0 \%) \text { Lack of leisure activities } \\
5(41.7 \%) \text { Staying inside } \\
5(41.7 \%) \text { Washing hands more } \\
3(25.0 \%) \text { Virtual care } \\
3(25.0 \%) \text { Limited contact with others (staff and clients) }\end{array}$ \\
\hline $\begin{array}{l}\text { What coping strategies did you use during the } \\
\text { outbreak, if any? }\end{array}$ & $\begin{array}{l}8(66.7) \text { Other (please specify) } \\
7(58.3 \%) \text { Leisure activities (e.g., reading, games, listen to music) } \\
3(25.0 \%) \text { Mindfulness/relaxation/meditation } \\
2(16.7 \%) \text { Ask for help when needed } \\
0(0.0 \%) 1: 1 \text { therapy with clinicians }\end{array}$ \\
\hline $\begin{array}{l}\text { Did the COVID droplet and contact precautions } \\
\text { change your relationships with clinicians on the } \\
\text { unit? }\end{array}$ & $\begin{array}{l}4(36.4 \%) \text { Yes } \\
7(63.3 \%) \text { No } \\
1(8.3 \%) \text { Did not respond }\end{array}$ \\
\hline $\begin{array}{l}\text { Are there things you would like to see done } \\
\text { differently during outbreak precautions? }\end{array}$ & $\begin{array}{l}7(63.6 \%) \text { Yes } \\
3(27.3 \%) \text { No } \\
1(9.1 \%) \text { Indifferent } \\
1(8.3 \%) \text { Did not respond }\end{array}$ \\
\hline $\begin{array}{l}\text { Do the restrictions need to be adapted to better } \\
\text { meet your needs? }\end{array}$ & $\begin{array}{l}10(90.9) \text { Yes } \\
1(9.1 \%) \text { No } \\
1(8.3 \%) \text { Did not respond }\end{array}$ \\
\hline
\end{tabular}

being offered on the units were reduced or paused during the COVID-19 outbreak. Clinicians reported, "Most of the group programs in and off the unit has been cancelled" (survey). "Less activity/programs, if there is a program it will be restricted" (survey). Another factor clinicians identified as affecting programming was limited access to resources to run the activities. "A lot of interventions listed we don't have enough (supplies) to give a single one to each 
Table 4: Patient and staff qualitative survey results

\begin{tabular}{|c|c|c|}
\hline Theme & Generic categories & Subcategories \\
\hline \multirow[t]{5}{*}{$\begin{array}{l}\text { Barriers to quality of } \\
\text { care during COVID-19 } \\
\text { outbreak precautions } \\
\text { on forensic units }\end{array}$} & $\begin{array}{l}\text { Decreased well-being and } \\
\text { quality of life of patients on } \\
\text { the unit }\end{array}$ & $\begin{array}{l}\text { Change in living conditions } \\
\text { The need to isolate patients in their rooms } \\
\text { Decreased socialization among patients and with unit staff } \\
\text { Restrictions on services outside the unit } \\
\text { Paused progression on patient goals }\end{array}$ \\
\hline & $\begin{array}{l}\text { Droplet and contact (D\&C) } \\
\text { precautions }\end{array}$ & $\begin{array}{l}\text { Patient disorganization } \\
\text { Mask compliance } \\
\text { Testing procedures }\end{array}$ \\
\hline & $\begin{array}{l}\text { Tension between clinical } \\
\text { staff, as well as between } \\
\text { patients and clinicians }\end{array}$ & $\begin{array}{l}\text { Tension between patients and clinicians } \\
\text { Tension between clinicians } \\
\text { Tension due to increased stress and staff burnout }\end{array}$ \\
\hline & $\begin{array}{l}\text { Limited resources } \\
\text { experienced by patients and } \\
\text { clinicians }\end{array}$ & $\begin{array}{l}\text { Access to PPE (personal protective equipment) } \\
\text { Sufficient staffing } \\
\text { Resources for patient programming }\end{array}$ \\
\hline & Leadership barriers & $\begin{array}{l}\text { Communication challenges } \\
\text { Organizational support challenges } \\
\text { Delays in implementation of supports } \\
\text { Changing COVID policies }\end{array}$ \\
\hline \multirow[t]{4}{*}{$\begin{array}{l}\text { Supportive aspects } \\
\text { of quality care during } \\
\text { COVID-19 outbreak } \\
\text { precautions on } \\
\text { forensic units }\end{array}$} & $\begin{array}{l}\text { More time spent in rooms } \\
\text { allowing patients to } \\
\text { participate in individual } \\
\text { activities }\end{array}$ & $\begin{array}{l}\text { Relaxation } \\
\text { Increased attendance to ADLs (activities of daily living) }\end{array}$ \\
\hline & $\begin{array}{l}\text { Patients enjoyed electronic } \\
\text { device access }\end{array}$ & $\begin{array}{l}\text { Access to tablets with streaming subscription } \\
\text { Access to electronic services }\end{array}$ \\
\hline & Supportive team dynamics & $\begin{array}{l}\text { Promoting teamwork and collaboration within the unit } \\
\text { Support from other units within the hospital }\end{array}$ \\
\hline & $\begin{array}{l}\text { Patient and staff dedication } \\
\text { to safety }\end{array}$ & $\begin{array}{l}\text { Understanding the need for safety precautions } \\
\text { Preventing spread of COVID-19 }\end{array}$ \\
\hline \multirow{4}{*}{$\begin{array}{l}\text { Changes desired to } \\
\text { improve quality of } \\
\text { care in future outbreak } \\
\text { precautions on } \\
\text { forensic units }\end{array}$} & $\begin{array}{l}\text { Focus on promoting patient } \\
\text { well-being and quality of life }\end{array}$ & $\begin{array}{l}\text { Decreased use of isolation } \\
\text { Increased access to outside services } \\
\text { Increased activities and services }\end{array}$ \\
\hline & $\begin{array}{l}\text { Improved access to and } \\
\text { cleanliness of bathrooms on } \\
\text { the unit }\end{array}$ & $\begin{array}{l}\text { Cleanliness of bathrooms } \\
\text { Access to bathrooms/waiting times } \\
\text { Staffing for bathrooms }\end{array}$ \\
\hline & $\begin{array}{l}\text { Increased access to } \\
\text { resources for clinicians and } \\
\text { patients }\end{array}$ & $\begin{array}{l}\text { Ensuring sufficient staffing } \\
\text { Access to Sufficient PPE }\end{array}$ \\
\hline & Proactive leadership & $\begin{array}{l}\text { Better communication from executives to team } \\
\text { Better communication between staff and patients }\end{array}$ \\
\hline
\end{tabular}

client" (survey). Clinicians shared their concerns with the restricted programming and the impact it had on their experience, stating, "We couldn't run any in-person groups and taking on the virtual groups was challenging. Types of groups we are able to provide are not the same as we did and it's met with a lot of disappointment from clients" (focus group). The impact of restricted programming was felt heavily by patients on the units, some stating they "couldn't do anything" (survey), and that the experience was "boring" (survey). One patient reflected on their disappointment with being unable to access the outdoor programming during the lockdown 
period, "It was hard to not even go to the yard. If you don't have COVID, you shouldn't be penalized like that" (survey).

Due to the restrictions in programming on and off the units, patients and staff felt a strain on socialization as well. As individuals needed to stay within their respective rooms, the socialization between patients was greatly reduced. Clinicians noted, "some patients usually interact with each other on a regular basis which was paused during the lockdown." and "Social life on the unit has taken a big negative impact as clients are discouraged from gathering" (survey). Another factor that impacted socialization on the units was the use of masks and face shields. Efficient and meaningful communication was impeded as it was challenging to hear through masks and face shields and nonverbal cues such as facial expressions are not possible.

Patients also felt the restriction in access to outside services and the community. Patients and clinicians both noted patients were "unable to get items (clothing or food)" and "passes are greatly restricted" (survey). This reduced access to the community has also led to paused progression to discharge for some clients. One patient explained how they were "almost going to work and then it got all pulled away. I almost had a landscaping job and COVID kept that from happening" (survey). A clinician expressed, "holding back their progress is what it felt like" (survey).

The D\&C precautions themselves were seen as challenging for patients. "Client disorganization made it more difficult to adhere to basic (D\&C) precaution rules without constant prompting (i.e., mask-wearing, sanitizing hands at different points while on a pass)" (survey). Many clinicians described the challenge of patients requiring constant cues to follow the D\&C protocols. "Numerous times that staff has to run and tell clients to wear their masks, clients who refuse to wear masks or refuse to go to their rooms when they are in common areas-issues with clients following precautions" and "Clients are really having a hard time wearing masks and starting to see increased frustrations, aggressions with clients not following directions with mask-wearing at this point" (focus group).

There was also a clear consensus that the testing procedures were generally a negative experience for the clients. One clinician reported, "Some clients were not prepared for what it would be like (painful), and this may have contributed to a lot of paranoia" and, "One client said, 'it felt like they were stabbing me in the brain"” (focus group).

A common theme that arose from the data was the tension felt between clinical staff, as well as between patients and clinicians on the unit. There was "more of a divide between the allied health staff and nursing staff. Sometimes the allied health staff would advocate for a bit more choice for the clients and the nursing staff would say 'oh no, that's too risky"' (focus group). Another clinician went on to explain "People have a lot of really really strong opinions and its really hard to navigate. And often some people come from a safety perspective and allied health comes from a quality-of-life perspective and it results in a lot of tension and conflicts" (focus group). "Another thing that impacted it [therapeutic rapport] was the lack of consistency as well. Some people were more lenient with things like wearing masks and other people aren't ... it totally ruined my rapport with the client because I am here enforcing it and others aren't" (focus group). Clinicians found themselves having to focus more on enforcing the rules, which was often met with a lot of disappointment and frustration from clients. "As staff it was constantly telling them 'Put your mask on, 'No stay in your room.' I could tell they were frustrated, and it was a long time, but as safety and as staff we had to reinforce safety of the protocols" (focus group). "I feel like the COVID police and a lot of times that's what majority of the interaction is based on... it has been very damaging" (focus group). Patients also endorsed feeling this tension, stating, "Some of the staff were not patient enough" (survey).

Both patients and staff also identified challenges in accessing sufficient resources as an added 
stressor to their experiences during the D\&C precautions. Clinicians experienced reduced access and availability of PPE when the outbreaks first occurred, and staff felt as though their requests for additional PPE were being questioned. "We have to beg-feels like we are stealing" (focus group) and "Especially with how often we use it. We were being questioned as to why they required more PPE" (focus group).

Staff were also affected by reduced staffing resources "Another challenge during outbreak was being short-staffed and having a level of anxiety" (focus group). "It was extremely stressful and people calling in sick, so we were understaffed for the first little bit too. So, having to handle all of these changes and additional cleaning duties .... So that was really challenging" (focus group). Many clinicians agreed with these statements, with some of the more senior staff mentioning that they had not experienced short-staffing and its consequences this badly at any point in their careers.

The most frequently reported obstacle clinicians faced was communication challenges from leadership to front-line staff. Clinicians reported "miscommunications and lack of clarity" (focus group) and how it served as an additional divide between clients and clinicians. "Every unit is doing things differently-clients see that and hear that and ask why they are allowed to do things on other units, but they are not-this causes a lot of frustration and also ruined therapeutic rapport between them and clients" (focus group). A consistent comment throughout all three focus group sessions was that staff logistical questions regarding things like family visits, family supply drop-offs, and food services, went unanswered for too long. One clinician noted, "We had questions about if clients can receive/ open parcels to them, families can drop off items for their loved ones, didn't get anything about that" (focus group). Another clinician stated that they "had a lot of questions and things weren't already in place-probably because this is the first time that it happened" (focus group). Inconsistent or insufficient communication about new protocols between services also led to logistical challenges, for example with mealtimes. One clinician reported "We didn't know dietary staff wasn't allowed to come on during the outbreak ... messaging was inconsistent ... everything was supposed to be disposable but there were times that things weren't, and we didn't know what we were supposed to do with them" (focus group).

\section{Supportive aspects of care}

The main enablers to patient care were grouped in the following generic categories:

1. more time spent in rooms allowing patients to participate in individual activities,

2. patients enjoyed electronic device access,

3. supportive team dynamics, and

4. dedication to safety.

The most-reported enabler of patient care was the dedication to the safety of both patients and clinicians. Although patients found it difficult to adhere to safety precautions and D\&C protocols, they understood that it was necessary for their safety. Patients stated, "it was hard but necessary" (survey) and "we have to follow public health" (survey), demonstrating some understanding of the policies' rationale. One patient explained, "It's good that they did that if we all got sick it would have been more hectic. I have to learn to be more considerate, it felt like I was in trouble, but I wasn't. I think it was good overall because we didn't all get sick" (survey). A clinician also suggested, "because the whole unit was on lockdown it was easier for clients to understand because everyone was in their room" (survey). The implementation of D\&C precautions was perceived by patients as protecting them from the virus and was positively received. Two clinicians shared how they believed clients felt about the protocols. "Patients felt more protected from contracting COVID-19 under these precautions" (survey). "Clients believed that the measures implemented will help save their lives" (survey).

Some patients benefited from having more time to spend in their rooms as it allowed them to engage in individual activities that served them. For some patients, this meant they were 
able to pay more attention to their activities of daily living, such as self-care tasks, which they may normally neglect. Some patients expressed a preference for taking medications and eating meals in their room. "Everything's done for you, you get all your meals brought to you because you can't leave so it's like room service" (survey). Lastly, some patients indicated staying in their rooms was not difficult for them, stating they had "more time to relax" (survey) and had "less responsibility" (survey). Some clients also endorsed the increased time in their rooms was n'ot a big change for them. "I typically spend a lot of time in my room, so I was used to this" (survey).

Another enabler to patient care was the availability and access to electronic devices and services. Many patients completing the survey reported that having a tablet with access to a streaming subscription was the factor that "made it easier" (survey), as it gave them something to do while being isolated in their rooms. Clinicians noticed the difference as well, one stating, "once we had [the tablets] in place they really enjoyed using them. They watched a lot of Netflix and actually had something to do in their rooms" (survey). Tablets were also used for virtual programming when possible. A clinician noted, "clients were able to do some groups available virtually which was nice in their rooms" (survey). In addition to the tablets, clients also had supervised access to make phone calls, to allow for some socialization in a time where visitors were prohibited.

Supportive team dynamics was another enabler to patient care identified by clinicians throughout the outbreaks. Some units found team unity was high, and it created a supportive environment for patients. One clinician explained how "It was interesting to see how cohesively the team came together to support our clients, it did not matter what disciplines we were from, we took on the responsibility to care for our patients" (survey). Others expressed that although it was a difficult time, the team united to provide clients with the care they needed. "We did work as a team despite all the difficulties" (survey), "worked as a team to get through it" (survey) and the "team worked well together" (survey). Furthermore, some clinicians found that there was support from other departments in the hospital. One clinician explained how they needed more PPE and other units offered them supplies.

\section{Suggestions for changes to improve quality of care in the event of a future outbreak}

The main changes needed to improve patient care were grouped into four generic categories:

1. the need to focus on promoting client well-being and quality of life,

2. improved access to and cleanliness of bathrooms on the unit,

3. increased access to resources for clinicians and clients, and

4. better leadership from $\mathrm{CAMH}$ as an organization.

As identified in the barriers, one of the biggest challenges that clinicians and patients faced during the outbreak was the reduction in programming. Going forward, clinicians and patients suggested that there should be an increase in or maintenance of activities and services that are readily available to them during an outbreak. Both patients and clinicians noted that these services should not affect individuals who do not test positive for COVID on the units, one patient stating, "it was hard to not even go to the yard if you don't have COVID you shouldn't be penalized like that" (survey). Finding new ways to still engage clients on the unit should be a priority for inpatient teams. Clinicians stated that we need "ways to engage clients who are in quarantine and keep them active and goal-focused. Having daily breaks for exercise out of their room is necessary" (survey). "Accommodations to safely support client activities outside of their room (but on the unit), i.e., use of the activity room or lounges for recreation" (survey).

The confinement felt by patients was a contributing factor to reduced mental health and well-being. Patients proposed simple changes to improve their well-being, like "Being able to 
leave my room at least once a day" (survey), "Somehow order out, hospital meals you don't like you have to eat and that sucks" (survey). "They should let the patients go to the community once a day with staff for shopping so they can get what they want and bring it back" (survey). While these pose logistical challenges while adhering to D\&C protocols, the needs identified could potentially be met with accommodations. A strong suggestion made by several clinicians was access to supportive counselling for patients and staff during this time to improve overall well-being. A strong statement from one occupational therapist noted the importance of supportive counselling for this group noting, "Specialized supportive counselling for what they are enduring .... This has been frustrating and traumatizing for them" (survey).

Another major theme that arose from the data was the challenges related to the washrooms on the unit. Many patients noted the need for "enhanced cleaning of the [shared] washrooms" (survey). One patient commented, "I had to wait for the bathroom to be unlocked and for water because staff were busy" (survey). Solutions were identified to address this, including "Everyone should get a call bell and staff should respond quickly because sometimes you need to go to the washroom urgently" (survey). Staff also saw the challenges, stating "We tried our best but there were times when they would use the washroom and someone else got in. If we were to do this again and we had era cleaning staff dedicated to making sure everything was cleaned thoroughly" (focus group) and "One of the barriers in my experience was we were supposed to clean washrooms every time clients would use it, and this was resource-intensive because someone had to be there for them to finish, leave the washroom so that you would clean it. .... Ideally, we would have hired outside for that position, watching the washrooms, so that we can sanitize it" (focus group).

Another direction for change identified was increased access to resources. Some staff identified the need for additional staffing specifically for ensuring sanitation of the units,
"Having an extra person assigned to sanitizing doors, sanitizing toilets, and all that would have been extremely appreciated" (focus group). While others suggested hiring more staff to ensure clients are appropriately supported, "Providing enough staffing will help to increase staff presence around the patient area to enhance reassurance and support" (survey), and "we often did not have enough staff willing to take clients out regularly to accommodate such changes" (survey). Patients also felt this same need, with one client stating, "Hire more staff" (survey). Ensuring sufficient staffing levels is not only necessary to meet the needs of clients on the unit, but also to reduce the likeliness of staff burnout during these difficult circumstances. "Working 12 hours, on an outbreak unit can be very difficult. If there is any way of increasing support with more staff, it would very much be appreciated" (survey).

Clinicians also identified a need for a streamlined process of obtaining PPE. Clinicians noted obtaining the PPE was an issue. "Shortages of PPE requiring lots of reordering" (survey). "Stores was very difficult in providing us with PPE that we needed. We had manager support but should not have to fight for PPE, biohazard boxes, etc. Someone should deliver these PPE when needed instead of us having to go to the stores" (survey).

Lastly, an overarching direction for change present in the data was the need for more proactive leadership and communication. Two communication streams needing improvement were identified:

\section{1. between leadership and the team and}

2. between staff and clients.

Clinicians identified a need for better communication among clinicians and leadership about the protocols to be implemented and followed. Clinicians requested "Increased communication about changes and supplying more resources" (survey) and "More proactive communication to staff about expectations/ requirements for managing potential staff exposure" (survey). Clinicians also identified 
wanting more acknowledgement and appreciation from leadership about their work during an outbreak. "Lack of appreciation for staff. Especially with some staff not receiving pandemic pay ... it created a lot of tension" (survey). "There was no celebration of the staff that had been working hard" (survey).

Clinicians also noted that they saw a need for better communication with clients about expectations and rules for lockdown, "Clearer communication to clients about expectations. Perhaps a written letter from management" (survey). One patient highlighted the importance of providing advanced warning for patients when possible, stating it would be good to "know ahead of time if we're going to be locked down so we can prepare" (survey).

\section{Discussion}

Our study is novel as it seeks recent patient and staff feedback of the experience of the lockdown conditions imposed by public health regulations due to COVID-19 outbreaks in minimum secure settings in old buildings with limited options for patients and staff. We found important themes emerging about how this is communicated and experienced. First, frustrations including obstruction to getting fresh air and limited bathroom access secondary to shared spaces requiring cleaning between uses were prominent. Second, access to PPE was at times difficult to achieve. Third, communication from both leadership (public health and forensic) and within the team to achieve consistency is of crucial importance. Fourth, finding other activities to replace programming and passes was important to lessening the losses imposed by the public health requirements. Due to the physical design of the inpatient forensic units, enforcement of D\&C precautions posed additional challenges than more modern forensic facilities might have faced (and indeed than more modern parts of our facility).

The strengths of the study include that data triangulation through both surveys and focus groups supported data saturation, with no new data, no new themes, and no new coding available for the units surveyed [7]. We were able to collect client and staff responses for six weeks starting one-month post unit outbreak. Collecting data soon after the outbreaks potentially minimized the impact of recall bias, improving the validity of responses [8].

The front-line reports of staff and patients in our sample echo and expand on the challenges of outbreak in other mental health settings as identified in recent studies [4,9-14]. This suggests that while the changes to care and difficulties therein are known, specific strategies and resources to overcome these difficulties are not readily available to front-line staff. Our team compiled the results of our survey with requested resources to create a tangible, easyto-access document for clients, clinicians, and managers to support care delivery and wellness during an outbreak. We also used this document to reflect the anonymized information we received directly back to the teams, providing an opportunity for clinicians to hear the client's experience in their own words, and for managers to hear clinicians' experiences through direct quotes. Clear action points were included for clinicians and managers to improve the quality of care and ease of access to the various resources available to support them.

This survey of patients and staff on inpatient forensic units following a COVID-19 outbreak supports the acceptance of D\&C precautions to manage spread while highlighting potential enablers, adaptations, and logistical changes that support patient care as well as patient and staff wellness during an outbreak.

There were several limitations to our study. The survey did not include any validated measures of stress. The sample was largely a convenience sample of staff and patients willing to complete the survey. The $32.4 \%$ response rate for clients and $38.5 \%$ for clinicians are low. Ss such, the data may be influenced by response bias. Social desirability, psychosis, and cognition may have influenced patients' responses given the nature of the setting. Although the interviews occurred from one month after the outbreak, recall bias may have been an issue. 
Acknowledgements: This paper was submitted on behalf of the CAMH Inpatient Forensic Outbreak Guidance Document team comprised of Shawnna Balasingham, Courtney Brennan, Sean Kidd, Dana Moyer, Patti Socha, Sabrina Umbrello, and Treena Wilkie.

\section{Conflict of Interest: None}

\section{References}

1. Simpson AIF, Chatterjee S, Darby P, Jones RM, Maheandiran M, Penney SR, Wilkie T. Management of COVID-19 response in a secure forensic mental health setting: Réponse à la gestion de la COVID-19 dans un établissement sécurisé de santé mentale et de psychiatrie légale. Can J Psychiatry. 2020 Oct;65(10):695-700. https://doi.org/10.1177/0706743720935648.

2. Lemieux AJ, Dumais Michaud AA, Damasse J, Morin-Major JK, Nguyen TN, Lesage A, Crocker AG. Management of COVID-19 for persons with mental illness in secure units: A rapid international review to inform practice in Québec. Victims \& Offenders. 2020;15(7-8):1337-1360. https://doi.org/10.1080/15564886.2020.1827111.

3. Franke I, Büsselmann M, Streb J, Dudeck M. Perceived institutional restraint is associated with psychological distress in forensic psychiatric inpatients. Front Psychiatry. 2019 June 11;10:410. https://doi.org/10.3389/fpsyt.2019.00410.

4. Tomlin J. What does social distancing mean for patients in forensic mental health settings? Forensic Sci Int Mind Law. 2020;1:100018. https://doi.org/10.1016/j.fsiml.2020.100018.

5. Ontario Agency for Health Protection and Promotion (Public Health Ontario). Best practices for managing COVID-19 outbreaks in acute care settings. Toronto, ON: Queen's Printer for Ontario; 2021.

6. Elo S, Kyngäs H. The qualitative content analysis process. J Adv Nurs. 2008 Apr;62(1):107-115. https://doi.org/10.1111/j.1365-2648.2007.04569.x.

7. Fusch PI, Ness LR. Are we there yet? Data saturation in qualitative research. The Qualitative Report. 2015;20(9):1408-1416. https://doi.org/10.46743/2160-3715/2015.2281.
8. Crosswell AD, Lockwood KG. Best practices for stress measurement: How to measure psychological stress in health research. Health Psychology Open. 2020:7(2)1-12. https://doi.org/10.1177/2055102920933072.

9. Billings, J, Greene T, Kember T, Grey N, El-Leithy S, Lee D, et al. Supporting hospital staff during COVID-19: Early interventions. Occupational Medicine. 2020 July;70(5):327-329. https://dx.doi. org/10.1093\%2Foccmed\%2Fkqaa098.

10. Boland X, Dratcu L. COVID-19 and acute inpatient psychiatry: The shape of things to come. Int JPsychiatry Clin Pract. 2020 June;25(2):132-134. https://doi.org/10.1080/13651501.2020.1801755.

11. Dieset I, Løvhaug L, Selle M, Kolseth A, Smeland OB, Færden A. Lessons learned from a cross-sectional survey among patients and staff in an acute psychiatric unit during an ongoing pandemic outbreak. Psychiatry Res. 2021 Apr;298:113779. https://doi.org/10.1016/j.psychres.2021.113779.

12. Hao F, Tan W, Jiang L, Zhang L, Zhao X, Zou $Y$, et al. Do psychiatric patients experience more psychiatric symptoms during COVID-19 pandemic and lockdown? A case-control study with service and research implications for immunopsychiatry. Brain Behav Immun. 2020 Jul;87:100-106. https://doi.org/10.1016/j.bbi.2020.04.069.

13. Soh KC, Khanna R, Parsons A, Visa B, Ejareh Dar M. Masks in Melbourne: $n$ inpatient mental health unit's COVID-19 experience. Australas Psychiatry. 2021 Apr;29(2):240-241. https://doi.org/10.1177/1039856220968394.

14. Wu D, Jiang C, He C, Li C, Yang L, Yue Y. Stressors of nurses in psychiatric hospitals during the COVID-19 outbreak. Psychiatry Res. 2020 Jun;288:112956.

https://doi.org/10.1016/j.psychres.2020.112956.

\section{Corresponding author}

Courtney Brennan, Centre for Addiction and Mental Health, Complex Care and Recovery Program-Forensic Division, University of Toronto, Department of Occupational Science and Occupational Therapy, 1001 Queen St. W., Unit 1-1, \#112, Toronto, ON M6J 1H1 Canada; courtney.brennan@camh.ca 